International Journal of Applied Mathematical Research, $5(2)(2016)$ 103-106
International Journal of Applied Mathematical Research
SPC
Website: www.sciencepubco.com/index.php/IJAMR
doi: $10.14419 /$ ijamr.v5i2.5988
Research paper

\title{
Solving three-dimensional Volterra integral equation by the reduced differential transform method
}

\author{
Abdelhalim Ziqan ${ }^{1 *}$, Sawsan Armiti ${ }^{1}$ and Iyad Suwan ${ }^{1}$ \\ ${ }^{1}$ Department Of Mathematics and Statistics, The Arab American University, Jenin, Palestine. \\ *Corresponding author E-mail:abdelhalim.ziqan@aauj.edu
}

\begin{abstract}
In this article, the results of two-dimensional reduced differential transform method is extended to three-dimensional case for solving three dimensional Volterra integral equation. Using the described method, the exact solution can be obtained after a few number of iterations Moreover, examples on both linear and nonlinear Volterra integral equation are carried out to illustrate the efficiency and the accuracy of the presented method.
\end{abstract}

Keywords: Volterra integral equation, Differential transform, Reduce differential transform.

\section{Introduction}

Many problems in many branches of science such as engineering, physics and other disciplines can be modeled as a Volterra integral equation of the second kind. In literature, different methods have been used to solve one and two-dimensional Volterra integral equation see for example $[2,7,10,11,14]$ and the references in $[3,13]$. In the recent years, many researchers studied Volterra integral equation using the differential transform method (DTM) $[1,5,6,9]$. For three-dimensional integral equations, in [4] the author used the three-dimensional differential transform method and authors in [8] applied the block-pulse functions methods on three-dimensional nonlinear mixed Volterra-Fredholm integral equation. Recently, the differential transform method is modified to the so-called Reduced Differential Transform Method to solve Volterra integral equation[1].

The aim of the presented article is to solve three-dimensional Volterra integral equation using the reduced differential transform method. So, we consider the 3-dimensional Volterra integral equation of the form:

$u(x, y, t)=f(x, y, t)+\int_{t_{0}}^{t} \int_{y_{0}}^{y} \int_{x_{0}}^{x} K(x, y, t, \omega, v, \tau)[u(\omega, v, \tau)]^{m} d \omega d v d \tau$,

where $u(x, y, t)$ is the unknown function, $m$ is a positive integer and the functions $K$ and $f$ are analytic in the domain of interest.

\section{Reduced differential transform method}

In this section, we present basic definitions and operations of the reduced differential transform method, for more details see [12] and the references therein.

Now, assume that the function of three variables $w(x, y, t)$ can be written as a multiple of two functions as follows: $w(x, y, t)=F(x, y) G(t)$, then $w(x, y, t)$ can be represented as:

$w(x, y, t)=\sum_{i=0 l=0}^{\infty} \sum^{\infty} F(i, j) x^{i} y^{j} \sum_{k=0}^{\infty} G(k) t^{k}=\sum_{i=0 l=0}^{\infty} \sum_{k=0}^{\infty} \sum^{\infty} W(i, j) x^{i} y^{j} t^{k}$, where the function $W(i, j)=F(i, j) G(k)$ is called the spectrum of $w(x, y, t)$

Definition 2.1. Let $w(x, y, t)$ be an analytic function in the domain of interest, the reduced differential transform function is

$W_{k}(x, y)=\frac{1}{k !}\left[\frac{\partial^{k}}{\partial t^{k}} w(x, y, t)\right]_{t=t_{0}}$.

Definition 2.2. The differential inverse reduced transform of $W_{k}(x, y)$ is defined by

$w(x, y, t)=\sum_{k=0}^{\infty} W_{k}(x, y)\left(t-t_{0}\right)^{k}=\sum_{k=0}^{\infty} \frac{1}{k !}\left[\frac{\partial^{k}}{\partial t^{k}} w(x, y, t)\right]_{t=t_{0}}\left(t-t_{0}\right)^{k}$.

In fact, the function $w(x, y, t)$ can be written in a finite series as follows

$w_{n}(x, y, t)=\sum_{k=0}^{n} W_{k}(x, y)\left(t-t_{0}\right)^{k}+R_{n}(x, y, t)$,

the tail function $R_{n}(x, y, t)$ is negligibly small.

Table 1: Basic operations of the reduced differential transofrm method

\begin{tabular}{|c|c|}
\hline Original function & Reduced differential transformed \\
\hline$w(x, y, t)=u(x, y, t) v(x, y, t)$ & $W_{k}(x, y)=\sum_{j}^{k} U_{j}(x, y) V_{k-j}(x, y)$ \\
\hline$w(x, y, t)=\alpha u(x, y, t) \pm \beta v(x, y, t)$ & $W_{k}(x, y)=\alpha U_{k}(x, y) \pm \beta V_{k}(x, y)$ \\
\hline$\frac{\partial^{n}}{\partial t^{n}} u(x, y, t)$ & $\frac{(k+n) !}{k !} U_{k+n}(x, y)$ \\
\hline$\frac{\partial^{m+n+s}}{\partial x^{m} \partial y^{n} \partial t^{s}} u(x, y, t)$ & $\frac{(k+s) !}{k !} \frac{\partial^{m+n}}{\partial x^{m} \partial y^{n}} U_{k+s}(x, y)$ \\
\hline $\sin (\alpha x+\beta y+\omega t)$ & $\frac{\omega^{k}}{k !} \sin \left(\frac{\pi k}{2 !}+\alpha x+\beta y\right)$ \\
\hline $\cos (\alpha x+\beta y+\omega t)$ & $\frac{\omega^{k}}{k !} \cos \left(\frac{\pi k}{2 !}+\alpha x+\beta y\right)$ \\
\hline$e^{\alpha t}$ & $\frac{\alpha^{k}}{k !}$ \\
\hline$x^{m} y^{n} t^{q}$ & $\left\{\begin{array}{cc}x^{m} y^{n}, & k=q \\
0, & \text { otherwise }\end{array}\right.$ \\
\hline
\end{tabular}




\section{Main results}

Suppose that the functions $W_{k}(x, y), U_{k}(x, y), G_{k}(x, y)$ and $V_{k}(x, y)$ are the reduced differential transform functions of $w(x, y, t), u(x, y, t)$, $g(x, y, t)$ and $v(x, y, t)$ respectively.

Theorem 3.1. If $w(x, y, t)=\int_{0}^{t} \int_{0}^{y} \int_{0}^{x} u(z, \omega, \tau) d z d \omega d \tau$ then

$W_{k}(x, y)=\frac{1}{k} \int_{0}^{y} \int_{0}^{x} U_{k-1}(\omega, \tau) d \omega d \tau$.

Proof. The $k^{t h}$ partial derivative of the function $w(x, y, t)$ is $\frac{\partial^{k}}{\partial t^{k}} w(x, y, t)=\int_{0}^{y} \int_{0}^{x} \frac{\partial^{k-1}}{\partial t^{k-1}} u(z, \omega, t) d z d \omega$. The result can be easily deduced from equation (3).

Theorem 3.2. Let $w(x, y, t)=\int_{0}^{t} \int_{0}^{y} \int_{0}^{x} u(z, \omega, \tau) v(x, y, t) d z d \omega d \tau$ then The reduced differential transform function of $w(x, y, t)$ is

$W_{k}(x, y)=\frac{1}{k} \int_{0}^{y} \int_{0}^{x} \sum_{r=0}^{k-1} U_{r}(\omega, \tau) V_{k-r-1}(\omega, \tau) d \omega d \tau$.

Proof. From Leibnitz formula the $k^{\text {th }}$ partial derivative of $w(x, y, t)$ is

$\int_{0}^{y} \int_{0}^{x} \sum_{r=0}^{k-1}\left(\begin{array}{c}k-1 \\ r\end{array}\right) \frac{\partial^{r}}{\partial t^{r}} u(\omega, \tau, t) \frac{\partial^{k-r-1}}{\partial t^{k-r-1}} v(\omega, \tau, t) d \omega d \tau$,

table (1) and equation (3) yield the following equation

$$
\begin{aligned}
\left.\frac{\partial^{k}}{\partial t^{k}} w\right|_{t=0} & =\int_{0}^{y} \int_{0}^{x} \sum_{r=0}^{k-1}\left(\begin{array}{c}
k-1 \\
r
\end{array}\right) r !(k-r-1) ! U_{r} V_{k-r-1} d \omega d \tau \\
& =(k-1) ! \int_{0}^{y} \int_{0}^{x} \sum_{r=0}^{k-1} U_{r}(\omega, \tau) V_{k-r-1}(\omega, \tau) d \omega d \tau
\end{aligned}
$$

Theorem 3.3. Let $w(x, y, t)=h(x, y, t) \int_{0}^{t} \int_{0}^{y} \int_{0}^{x} u(z, \omega, \tau) d z d \omega d \tau$, then the reduced differential transform of $w(x, y, t)$ is

$W_{k}(x, y)=\sum_{r=0}^{k-1} \frac{1}{k-r} H_{r}(x, y) \int_{0}^{y} \int_{0}^{x} U_{k-r-1}(\omega, \tau) d \omega d \tau$.

Proof. The $k^{t h}$ partial derivative of $w(x, y, t)$ with respect to $t$ is

$$
\frac{\partial^{k}}{\partial t^{k}} w(x, y, t)=\sum_{r=0}^{k}\left(\begin{array}{l}
k \\
r
\end{array}\right) \frac{\partial^{r}}{\partial t^{r}} h(x, y, t) \int_{0}^{y} \int_{0}^{x} \frac{\partial^{k-r-1}}{\partial t^{k-r-1}} u(\omega, \tau, t) d \omega d \tau .
$$

On the other hand, $\left[\frac{\partial^{k-r}}{\partial t^{k-r}} \int_{0}^{t} \int_{0}^{y} \int_{0}^{x} u(z, \omega, \tau) d z d \omega d \tau\right]_{t=0}=0$ for $k=r$. Thus, from equation (3) we have $\left[\frac{\partial^{k}}{\partial t^{k}} w(x, y, t)\right]_{t=0}=k ! W_{k}(x, y)$ and hence,

$$
\left[\frac{\partial^{k}}{\partial t^{k}} w(x, y, t)\right]=\sum_{r=0}^{k-1}\left(\begin{array}{l}
k \\
r
\end{array}\right) r !(k-r-1) ! H_{r}(x, y) \int_{0}^{y} \int_{0}^{x} U_{k-r-1}(\omega, \tau) d \omega d \tau
$$

Theorem 3.4. If $w(x, y, t)=\int_{0}^{t} \int_{0}^{y} \int_{0}^{x} \frac{u(z, \omega, \tau)}{v(z, \omega, \tau)} d z d \omega d \tau$, then

$U_{k}(x, y)=\sum_{r=0}^{k}(r+1) \frac{\partial^{2} W_{r+1}(x, y)}{\partial x \partial y} V_{k-r}(x, y)$.

Proof. First write the function $u(x, y, t)=\frac{\partial^{3} w(x, y, t)}{\partial x \partial y \partial t} v(x, y, t)$ then we differentiate partially $k$ times with respect to $t$ to get the following equation

$$
\frac{\partial^{k}}{\partial t^{k}} u(x, y, t)=\sum_{r=0}^{k-1}\left(\begin{array}{l}
k \\
r
\end{array}\right) \frac{\partial^{r+3}}{\partial x \partial y \partial t^{r+1}} w(x, y, t) \frac{\partial^{k-r}}{\partial t^{k-r}} v(\omega, \tau, t) .
$$

Therefore, from equation (3) we have

$k ! U_{k}(x, y)=\sum_{r=0}^{k}(r+1) !(k-r) ! \frac{\partial^{2} W_{r+1}(x, y)}{\partial x \partial y} V_{k-r}(x, y)$.

Theorem 3.5. If $w(x, y, t)=\frac{1}{v(x, y, \tau)} \int_{0}^{t} \int_{0}^{y} \int_{0}^{x} u(z, \omega, \tau) d z d \omega d \tau$ then

$\sum_{r=0}^{k} W_{r}(x, y) V_{k-r}(x, y)=\frac{1}{k} \int_{0}^{y} \int_{0}^{x} U_{k-r}(\omega, \tau) d \omega d \tau$.

Proof. Write the given integral equation as

$w(x, y, t) v(x, y, \tau)=\int_{0}^{t} \int_{0}^{y} \int_{0}^{x} u(z, \omega, \tau) d z d \omega d \tau$

So, equation (10) can be easily obtained from equation (6) and table (1).

\section{Numerical examples}

In this section, we apply RDTM method on several examples of linear and nonlinear Volterra integral equation. Then we close this section by an example which is solved in[4] by DTM method and present the table of absolute error at some particular points to compare between the two methods.

Example 4.1. Consider the integral equation $u(x, y, t)=x \cos t-\frac{x^{3} y^{3}}{9} \sin t+\int_{0}^{t} \int_{0}^{y} z \omega_{0}^{2} u(z, \omega, \tau) d z d \omega d \tau$.

The exact solution is $u(x, y, t)=x \cos t$. First, let $f(x, y, t)=x \cos t-\frac{x^{3} y^{3}}{9} \sin t$ and $v(x, y, t)=x y^{2}$.

Elementary calculations lead to $F_{0}(x, y)=x, F_{1}(x, y)=-\frac{x^{3} y^{3}}{9}$, $F_{2}(x, y)=\frac{-x}{2}, F_{3}(x, y)=\frac{x^{3} y^{3}}{3 ! 9}, \ldots$ and $V_{0}(x, y)=x y^{2}, V_{k}(x, y)=0$ for $k \geq 1$. Also, $U_{0}(x, y)=F_{0}(x, y)=x$. Now, from theorem (3.2), $U_{k}(x, y)=F_{k}(x, y)+\frac{1}{k} \iint_{0}^{y} \int_{0}^{x} \sum_{r=0}^{k-1} U_{r}(\omega, \tau) V_{k-r-1}(\omega, \tau) d \omega d \tau, k \geq 1$.

So, easy calculation on the last integral equation will produce the following formula:

$U_{k}(x, y)=\left\{\begin{array}{cc}\frac{(-1)^{k} x}{k !}, & k \text { is even } \\ 0, & k \text { is odd }\end{array}\right.$

Therefore,

$u(x, y, t)=\sum_{k=0}^{\infty} U_{k}(x, y) t^{k}=x \sum_{k=0}^{\infty} \frac{(-1)^{k} t^{2 k}}{(2 k) !}=x \cos t$.

Example 4.2. Consider the nonlinear integral equation

$u(x, y, t)=x y t-\frac{(x y t)^{3}}{27}+\int_{0}^{t} \int_{0}^{y} \int_{0}^{x} u^{2}(z, \omega, \tau) d z d \omega d \tau$.

The exact solution to this integral equation is $u(x, y, t)=x y t$. 
It is clear that $U_{0}(x, y)=F_{k}(x, y)=0, k \neq 1$ and $F_{1}(x, y)=x y$. Also, from theorem(3.2) we have

$U_{k}(x, y)=F_{k}(x, y)+\frac{1}{k} \int_{0}^{y} \int_{0}^{x} \sum_{r=0}^{k-1} U_{r}(\omega, \tau) U_{k-r-1}(\omega, \tau) d \omega d \tau, k \geq 1$.

Hence $U_{1}(x, y)=x y$ and $U_{k}(x, y)=0$ for $k \neq 1$.

Example 4.3. Consider the following integral equation $u(x, y, t)=g(x, y, t)+w(x, y, t)$ where $v(x, y, t)=1+\cos t$ and

$$
\begin{aligned}
w(x, y, t) & =\int_{0}^{t} \int_{0}^{y} \int_{0}^{x} \frac{u(z, \omega, \tau)}{v(z, \omega, \tau)} d z d \omega d \tau, g(x, y, t) \\
& =(x+y)(1+\cos t)-\frac{x^{2} y+x y^{2}}{2}
\end{aligned}
$$

It is obvious that $U_{0}(x, y)=G_{0}(x, y)=2(x+y)$. Also, from table (1), $G_{1}(x, y)=-\frac{x^{2} y+x y^{2}}{2}$, and for $k \geq 2$

$G_{k}(x, y)=\left\{\begin{array}{cc}(-1)^{k+1}(x+y), & k \text { even } \\ 0 & k \text { odd }\end{array}\right.$

On the other hand, $V_{0}(x, y)=2$ and for $k \geq 1$,

$V_{k}(x, y)=\left\{\begin{array}{cc}\frac{1}{k !} \cos \left(\frac{k \pi}{2}\right), & k \text { even } \\ 0, & k \text { odd }\end{array}\right.$

Using (9), $W_{1}(x, y)=\frac{x^{2} y+x y^{2}}{2}, W_{2}(x, y)=0$. But since

$U_{k}(x, y)=G_{k}(x, y)+W_{k}(x, y)$ we have for $k \geq 1$,

$U_{k}(x, y)=\left\{\begin{array}{cc}\frac{-(x+y)}{k !}, & k \text { even } \\ 0 & k \text { odd }\end{array}\right.$

Therefore,

$$
\begin{aligned}
u(x, y, t) & =2(x+y)+\frac{-(x+y)}{2} t^{2}+\frac{(x+y)}{4 !} t^{4}+\cdots \\
& =(x+y)\left[1+\left(1+\frac{-1}{2} t^{2}+\frac{1}{4 !} t^{4}+\cdots\right)\right] \\
& =(x+y)(1+\cos t) .
\end{aligned}
$$

Example 4.4. Consider the following integral equation $u(x, y, t)=g(x, y, t)+\int_{0}^{t} \int_{0}^{y} \int_{0}^{x} u(z, \omega, \tau) d z d \omega d \tau$, where $0 \leq x, y, t \leq 1$ and $g(x, y, t)=e^{x+y}+\left(e^{t}-1\right)\left(e^{x}+e^{y}-1\right)$. The exact solution is $u(x, y, t)=e^{x+y+t}$.

It is clear that $U_{0}(x, y)=G_{0}(x, y)=e^{x+y}$. Also, from table (1) , $G_{k}(x, y)=\frac{1}{k !}\left(e^{x}+e^{y}-1\right)$. Theorem (3.1) implies

$U_{k}(x, y)=G_{k}(x, y)+\frac{1}{k} \int_{0}^{y} \int_{0}^{x} U_{k-1}(\omega, \tau) d \omega d \tau, k=1,2, \ldots$

Using equation (11) recursively one can obtain the general formula of $U_{k}(x, y)=\frac{e^{x+y}}{k !}$ and hence $u(x, y, t)=\sum_{k=0}^{\infty} \frac{e^{x+y}}{k !} t^{k}=e^{x+y+t}$.

The exact solution and absolute error of the iterative solutions $u_{2}(x, y, t)$ and $u_{3}(x, y, t)$ obtained by RDTM at some test points $(x, y, t)$ are calculated in tables(2) and (3). Comparing the results obtained by RDTM method with those obtained by DTM method (see example 3.3 [4]), we conclude that RDTM minimizes the number of iterations to reach the exact solution. Also,the approximated solution approaches rabidly to the exact solution.
Table 2: The Exact Solution

\begin{tabular}{c|c|c|c}
\hline$x$ & $y$ & $t$ & Exact Solution \\
\hline 0.1 & 0.1 & 0.1 & 1.3498588076 \\
0.01 & 0.1 & 0.1 & 1.2336780600 \\
0.01 & 0.01 & 0.1 & 1.1274968516 \\
0.01 & 0.01 & 0.01 & 1.0304545340 \\
0.001 & 0.01 & 0.01 & 1.0212220516 \\
0.001 & 0.001 & 0.01 & 1.0120722889 \\
0.001 & 0.001 & 0.001 & 1.0030045045 \\
\hline
\end{tabular}

Table 3: The Absolute Error

\begin{tabular}{c|c|c|c|c}
\hline$x$ & $y$ & $t$ & $\left|u_{\text {exact }}-u_{2}\right|$ & $\left|u_{\text {exact }}-u_{3}\right|$ \\
\hline 0.1 & 0.1 & 0.1 & $2.08759809 \times 10^{-4}$ & $5.19268266 \times 10^{-6}$ \\
0.01 & 0.1 & 0.1 & $1.90792099 \times 10^{-4}$ & $4.7457546 \times 10^{-6}$ \\
0.01 & 0.01 & 0.1 & $1.74370849 \times 10^{-4}$ & $4.3372931 \times 10^{-6}$ \\
0.01 & 0.01 & 0.01 & $1.7045949 \times 10^{-7}$ & $4.2594 \times 10^{-10}$ \\
0.001 & 0.01 & 0.01 & $1.6893224 \times 10^{-7}$ & $4.2212 \times 10^{-10}$ \\
0.001 & 0.001 & 0.01 & $1.6741867 \times 10^{-7}$ & $4.1834 \times 10^{-10}$ \\
0.001 & 0.001 & 0.001 & $1.6704 \times 10^{-10}$ & $4 \times 10^{-15}$ \\
\hline
\end{tabular}

\section{Conclusion}

In this paper, the three dimensional linear and nonlinear integral equations are solved by using RDTM. It is worth noting that RDTM does not require complex computational work like DTM. It can be easily implemented, its convergence is rapid and its approximation is accurate. In general, it can be concluded that RDTM is a powerful tool for solving many linear and nonlinear three dimensional integral equations.

\section{Acknowledgement}

The authors would like to thank Mr. Hayel Al-Shraydeh for his support in improving the print of the manuscript.

\section{References}

[1] R. Abazari, A. Kiliçman "Numerical study of two-dimensional Volterra integral equation by RDTM and comparison with DTM", Abstr Appl Anal Volume 2013, (2013), doi.org/101155/2013/929478.

[2] G.A. Afroozi, J. Vahidi, M. Saeidy, "Solving a class of two-dimensional linear and nonlinear Volterra integral equation by means of the homotopy analysis method", Int J Nonlinear Sci, Vol.9 No.2, (2010) 213-219.

[3] E. Babolian, K. Maleknejad, M. Roodaki, H. Almasieh, "Twodimensional triangular functions and their applications to nonlinear 2D Volterra-Fredholm integral equations", Comput Math Appl, 60(6), (2010) 1711-1722

[4] M. Bakhshi, Mohhammad Asghari-Larimi, M. Asghari-Larimi, "Threedimensional differential transform method for solving nonlinear threedimensional Volterra integral equations", The Journal of Mathematics and Computer Science, Vol. 4 No.2, (2012), 246 - 256.

[5] N. Dogan, V. Erturk, S. Momani, O. Akin, A. Yildirim, "Differential transform method for solving singularly perturbed Volterra integral equations", Journal of King Saud University- science, 23(2), (2011) 223- 228.

[6] B. Jang, "Comments on solving a class of two-dimensional linear and nonlinear Volterra integral equations by the differential transform method", J Comput Appl Math, 233(2), (2009)224-230.

[7] R.P. Kanwal, Linear Integral Equations, Springer Science and Business Media, 2013.

[8] F. Mirzaee, E. Hadadiyan, S. Bimesl, "Numerical solution for threedimensional nonlinear mixed Volterra-Fredholm integral equations via three-dimensional block-pulse functions”, Appl Math Comput, 237, (2014) 168-175

[9] M. M. Moghadam, H.Saeedi, "Application of differential transform for solving the Volterra integro-partial equations", Iran J Sci Technol (Sciences), 34(1), (2010) 59-70.

[10] F. Mohammadi, "A Chebyshev wavelet operational method for solving stochastic Volterra-Fredholm integral equations", Int J Appl Math Research, 4 (2), (2015) 217-227.

[11] R. Rahman, Integral Equations and Their Applications, Wit Pr/Computational Mechanics, 2007. 
[12] V.K. Srivastava, M.K. Awasthi, R.K. Chaurasia,reduced differential transform method to solve two and three dimensional second order hyperbolic telegraph equations, Journal of King Saud University - Engineering Sciences, (2014), http://dx.doi.org/10.1016/j.jksues.2014.04.010.

[13] A. Tari, M.Y. Rahimi, S. Shahmorad, F. Talati, " Solving a class of two-dimensional linear and nonlinear Volterra integral equations by the differential transform method", J Comput Appl Math, 228(1), (2009) 70-76

[14] A.M. Wazwaz, Linear and nonlinear integral equations: methods and applications, Springer Science and Business Media, 2011. 